\title{
A Collimated Molecular Jet in W 43A Traced by Water Maser Emission
}

\author{
Hiroshi Imai and Tetsuo Sasao \\ VERA Project Office, National Astronomical Observatory, Japan
}

Kumiko Obara and Toshihiro Omodaka

Department of Physics, Kagoshima University, Japan

\author{
Philip J. Diamond \\ Jodrell Bank Observatory, University of Manchester, United Kingdom
}

\begin{abstract}
We present VLBA observations of the spatial and velocity distributions of $22 \mathrm{GHz} \mathrm{H} \mathrm{H}_{2} \mathrm{O}$ and $1612 \mathrm{MHz} \mathrm{OH}$ masers in the $\mathrm{OH} / \mathrm{IR}$ star $\mathrm{W} 43 \mathrm{~A}$. These masers have the same systemic velocity and are, therefore, likely to be associated with the common stellar object. However, the kinematical structures of them are quite different and independent. Most of the $\mathrm{H}_{2} \mathrm{O}$ masers are extremely collimated spatially and kinematically. The $\mathrm{H}_{2} \mathrm{O}$ maser jet also seems to be precessing . On the other hand, the $\mathrm{OH}$ masers exhibit clear arc-shaped structures indicating a sphericallyexpanding shell with weak collimation. The $\mathrm{W} 43 \mathrm{~A}$ jet is very likely to be predominantly composed of hot molecules traced by $\mathrm{H}_{2} \mathrm{O}$ maser emission and formed in the immediate vicinity of an unknown star next to another $\mathrm{OH} / \mathrm{IR}$ star. Such a "molecular jet" is likely to appear only during the short period before a star forms an elongated planetary nebula.
\end{abstract}

$\mathrm{H}_{2} \mathrm{O}$ and $\mathrm{OH}(1612 \mathrm{MHz})$ masers in $\mathrm{W} 43 \mathrm{~A}$ have been observed using the NRAO's VLBA on three occasions: 1994 June 25, 1994 October 10, and 1995 March 17. The obtained angular and velocity resolutions were 0.5 mas and 0.21 $\mathrm{km} \mathrm{s}^{-1}$ at $22.2 \mathrm{GHz}$ band and 9 mas and $0.36 \mathrm{~km} \mathrm{~s}^{-1}$ at $1.6 \mathrm{GHz}$ band, respectively. We measured proper motions of $21 \mathrm{H}_{2} \mathrm{O}$ maser features that were detected in two or three epochs. Figure 1 shows the kinematics of the $\mathrm{H}_{2} \mathrm{O}$ and $\mathrm{OH}$ masers.

Most of $\mathrm{H}_{2} \mathrm{O}$ masers in $\mathrm{W} 43 \mathrm{~A}$ are concentrated in blue-shifted and redshifted clusters, both of which are surprisingly spatially collimated with a width of only $20 \mathrm{AU}$. The two clusters have lengths of 250-350 AU and are separated by $1700 \mathrm{AU}$. The 3-D motions of the masers indicate a collimated, fast jet-like motion with a $3-\mathrm{D}$ velocity of $145 \mathrm{~km} \mathrm{~s}^{-1}$. In addition, the direction of the spatial alignments of the water masers in W $43 \mathrm{~A}$ is shifted slightly by about $10^{\circ}$ from both the direction of the cluster separation and the jet direction, implying that the jet is precessing. The observed spatial pattern is consistent with a model to consider a jet with a constant velocity of $150 \mathrm{~km} \mathrm{~s}^{-1}$, an inclination of $36^{\circ}$ with respect to the sky plane, a position angle of $63^{\circ}$, and an axis precession with an angular amplitude of $5^{\circ}$ and a period of 55 years. 


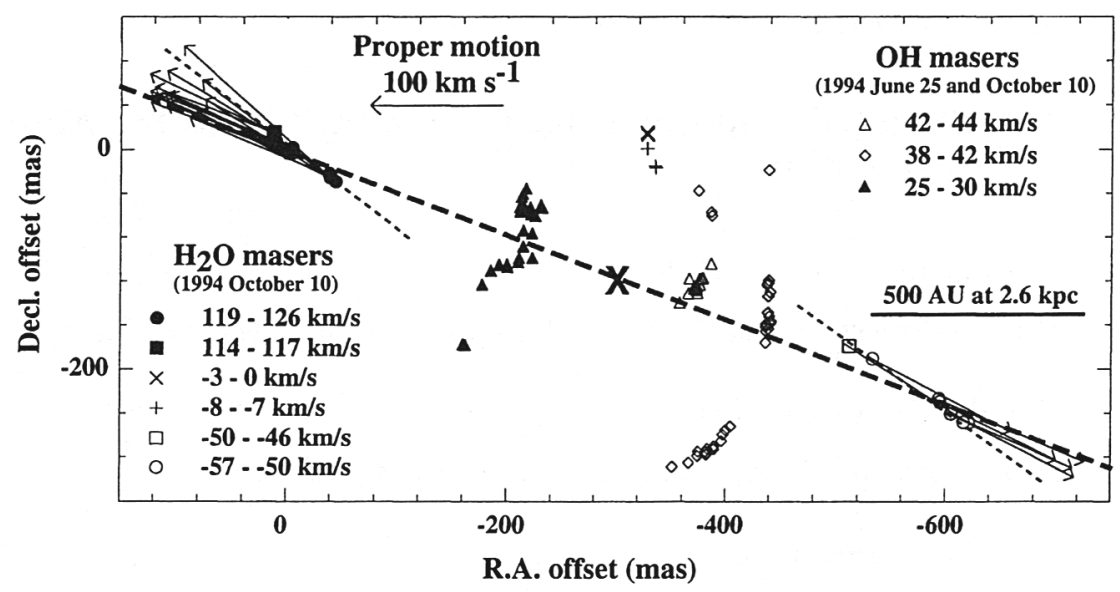

Figure 1. Kinematics of $\mathrm{H}_{2} \mathrm{O}$ and $1612 \mathrm{MHz} \mathrm{OH}$ masers in $\mathrm{W} 43 \mathrm{~A}$. Arrows show velocity vectors of $21 \mathrm{H}_{2} \mathrm{O}$ masers. A dashed line shows the direction of the jet at a position angle of $69^{\circ}$. Two dotted thin lines show the alignments of maser spots in the individual clusters. A cross shows the estimated location of the central object at $(\Delta \alpha=-296$ mas, $\Delta \delta=-112$ mas) with a systemic radial velocity of $V_{\mathrm{LSR}}=34 \mathrm{~km} \mathrm{~s}^{-1}$. The position offsets of the $\mathrm{OH}$ masers relative to the $\mathrm{H}_{2} \mathrm{O}$ masers were estimated by assuming the common central object at the middle point of the red-shifted and blue-shifted $\mathrm{OH}$ masers.

In contrast to $\mathrm{H}_{2} \mathrm{O}$ masers, $\mathrm{OH}$ masers in $\mathrm{W} 43 \mathrm{~A}$ have clear arc-shaped structures that can fit a model of a spherically-expanding shell with a radius of $\sim 500 \mathrm{AU}$ around the star with an expansion velocity of $9 \mathrm{~km} \mathrm{~s}^{-1}$ (e.g. Diamond and Nyman 1988). Usually the morphology and kinematics of $\mathrm{H}_{2} \mathrm{O}$ masers are complicated or elongated perpendicular to the directions of the outflows because $\mathrm{H}_{2} \mathrm{O}$ masers are excited around shocks between an outflow and the ambient gas cloud. In addition, $\mathrm{H}_{2} \mathrm{O}$ masers around an evolved star are located closer to the star than $\mathrm{OH}$ masers. From these results, we propose a model of $\mathrm{H}_{2} \mathrm{O}$ and $\mathrm{OH}$ masers in $\mathrm{W} 43 \mathrm{~A}$ as follows. The circumstellar envelope of $\mathrm{W} 43 \mathrm{~A}$ is destroyed at closer part from the star at final phase of the $\mathrm{OH} / \mathrm{IR}$ star stage. The $\mathrm{H}_{2} \mathrm{O}$ masers are excited only the tips of a highly-collimated jet with compact clumps simultaneously ejected from the star. Such a collimated jet originating from an $\mathrm{OH} / \mathrm{IR}$ star is surprising, but can be explained if $\mathrm{W} 43 \mathrm{~A}$ contains a binary system; an evolved star and another star generating the $\mathrm{OH}$ maser flow and the $\mathrm{H}_{2} \mathrm{O}$ maser jet with precession, respectively. The second star is likely to be a unique object that generates such a molecular jet in a short period of evolution prior to a white dwarf.

Acknowledgments. The NRAO is a facility of the National Science Foundation of USA, operated under a cooperative agreement by Associated Universities, Inc.

\section{References}

Diamond, P.J., \& Nyman, L.-A. 1988, in IAU Symp. 129, VLBI and their impact on Astrophysics, ed. J.M. Moran \& M.J. Reid (Dordrecht: Reidel), 249 